\title{
Performance of Plasma Amyloid $\beta$, Total Tau, and Neurofilament Light Chain in the Identification of Probable Alzheimer's Disease in South China
}

\section{OPEN ACCESS}

Edited by:

Nicholas James Ashton, University of Gothenburg, Sweden

Reviewed by:

Marta Mila Aloma

BarcelonaBeta Brain Research

Center, Spain

Fabricio Ferreira de Oliveira,

Elysian Clinic, Brazil

Alberto Benussi,

University of Brescia, Italy

${ }^{*}$ Correspondence:

Lu Shen

shenlu@csu.edu.cn; shenlu2505@126.com

tThese authors have contributed equally to this work

Received: 29 July 2021 Accepted: 24 September 2021 Published: 27 October 2021

Citation:

Jiao B, Liu H, Guo L, Liao X, Zhou Y, Weng L, Xiao $X$, Zhou L, Wang $X$,

Jiang $Y$, Yang $Q$, Zhu Y, Zhou L, Zhang $W$, Wang J, Yan $X$, Tang $B$ and Shen $L$ (2021) Performance of Plasma

Amyloid $\beta$, Total Tau, and Neurofilament Light Chain in the Identification of Probable Alzheimer's Disease in South China. Front. Aging Neurosci. 13:749649.

doi: 10.3389/fnagi.2021.749649
Bin Jiao ${ }^{1,2,3+}$, Hui Liu ${ }^{1 \dagger}$, Lina Guo ${ }^{1}$, Xinxin Liao ${ }^{2,3,4}$, Yafang Zhou ${ }^{2,3,4}$, Ling Weng 1,2,3, Xuewen Xiao ${ }^{1}$, Lu Zhou ${ }^{1}$, Xin Wang ${ }^{1}$, Yaling Jiang ${ }^{1}$, Qijie Yang ${ }^{1}$, Yuan Zhu ${ }^{1}$, Lin Zhou ${ }^{2,3,4}$, Weiwei Zhang $^{5}$, Junling Wang ${ }^{1,2,3}$, Xinxiang Yan ${ }^{1,2,3}$, Beisha Tang ${ }^{1,2,3}$ and Lu Shen ${ }^{1,2,3,6,7,8 *}$

${ }^{1}$ Department of Neurology, Xiangya Hospital, Central South University, Changsha, China, ${ }^{2}$ National Clinical Research Center for Geriatric Disorders, Central South University, Changsha, China, ${ }^{3}$ Key Laboratory of Hunan Province in Neurodegenerative Disorders, Central South University, Changsha, China, ${ }^{4}$ Department of Geriatrics, Xiangya Hospital, Central South University, Changsha, China, ${ }^{5}$ Department of Radiology, Xiangya Hospital, Central South University, Changsha, China, ${ }^{6}$ Key Laboratory of Organ Injury, Aging and Regenerative Medicine of Hunan Province, Changsha, China, ${ }^{7}$ Engineering Research Center of Hunan Province in Cognitive Impairment Disorders, Central South University, Changsha, China, ${ }^{8}$ Hunan International Scientific and Technological Cooperation Base of Neurodegenerative and Neurogenetic Diseases, Changsha, China

Background: Alzheimer's disease (AD) is the most common type of dementia and has no effective treatment to date. It is essential to develop a minimally invasive blood-based biomarker as a tool for screening the general population, but the efficacy remains controversial. This cross-sectional study aimed to evaluate the ability of plasma biomarkers, including amyloid $\beta(A \beta)$, total tau (t-tau), and neurofilament light chain (NfL), to detect probable $A D$ in the South Chinese population.

Methods: A total of 277 patients with a clinical diagnosis of probable $A D$ and 153 healthy controls with normal cognitive function $(\mathrm{CN})$ were enrolled in this study. The levels of plasma $A \beta 42, A \beta 40$, t-tau, and NfL were detected using ultra-sensitive immune-based assays (SIMOA). Lumbar puncture was conducted in 89 patients with AD to detect $A \beta 42, A \beta 40$, t-tau, and phosphorylated (p)-tau levels in the cerebrospinal fluid (CSF) and to evaluate the consistency between plasma and CSF biomarkers through correlation analysis. Finally, the diagnostic value of plasma biomarkers was further assessed by constructing a receiver operating characteristic $(\mathrm{ROC})$ curve.

Results: After adjusting for age, sex, and the apolipoprotein E (APOE) alleles, compared to the $\mathrm{CN}$ group, the plasma t-tau, and NfL were significantly increased in the AD group ( $p<0.01$, Bonferroni correction). Correlation analysis showed that only the plasma t-tau level was positively correlated with the CSF t-tau levels $(r=0.319, p=0.003)$. The diagnostic model combining plasma t-tau and NfL levels, and age, sex, and APOE alleles, showed the best performance for the identification of probable AD [area under the curve $(A \cup C)=0.89$, sensitivity $=82.31 \%$, specificity $=83.66 \%]$.

Conclusion: Blood biomarkers can effectively distinguish patients with probable AD from controls and may be a non-invasive and efficient method for AD pre-screening.

Keywords: Alzheimer's disease, plasma biomarkers, amyloid-beta, total-tau, neurofilament light chain 


\section{INTRODUCTION}

Currently, $\sim 50$ million people in the world are living with dementia and every $3 \mathrm{~s}$ a new case of dementia is diagnosed (Christina, 2018). Alzheimer's disease (AD) is the most common neurodegenerative dementia in older people and is characterized by progressive cognitive decline and behavioral defects with a complex and heterogeneous pathophysiology.

A preclinical phase of $\geq 20$ years may occur before the clinical diagnosis of $\mathrm{AD}$, during which no or only subtle symptoms appear, adding to the difficulty of early diagnosis and prevention (Jack et al., 2013; Jansen et al., 2015). At present, the most well-established AD biomarkers are mainly based on the core pathological features, including amyloid $\beta(\mathrm{A} \beta)$ deposition [detected by cerebrospinal fluid (CSF)] A $\beta 42$ levels, amyloid positron emission tomography (PET), neurodegeneration [CSF total tau (t-tau)] and phosphorylated (p)-tau levels, structural MRI, and hypometabolism on fluorodeoxyglucose (FDG)-PET (Desikan et al., 2009; Mattsson et al., 2009; Landau et al., 2012). Nevertheless, high invasiveness, expensive costs, and limited availability hinder their clinical application. Therefore, noninvasive, cost-effective, and easily accessible biomarkers are desperately needed for the identification of AD.

To date, many studies have focused on blood-based biomarkers for the diagnosis of $\mathrm{AD}$; somewhat promising results have mainly been achieved with $\mathrm{A} \beta$, tau, and neurofilament light chain (NfL) in the blood. Previous studies have described the excellent performance of plasma $\mathrm{A} \beta$-related peptides for the prediction of AD using different methods (Chen et al., 2019; Vergallo et al., 2019). The plasma t-tau level is a promising candidate marker because it is a brain-specific protein that is mainly expressed in central nervous system (CNS) neurons, indicates neuronal damage, and is derived from the brain parenchyma and transported to the CSF and blood (SchraenMaschke et al., 2008). It has also been shown to enhance the prediction of dementia and is suggested to serve as a biomarker for risk stratification in dementia prevention trials (Mielke et al., 2017). NfL, the main component of the axonal cytoskeleton, is mainly expressed in large-caliber myelinated axons and released into the CSF following neuroaxonal injury (Petzold, 2005). Recently, robust studies have shown that NfL level in the peripheral blood is a promising biomarker for tracking neurodegenerative changes in patients with $\mathrm{AD}$ and increased levels are related to brain atrophy, brain hypometabolism, and decreased cognitive function (Zetterberg et al., 2016; Mattsson et al., 2017; Mayeli et al., 2019). Due to the poor disease specificity, the clinical application in detecting $\mathrm{AD}$ is limited (Wilke et al., 2016; Bridel et al., 2019). Compelling and emerging evidence has highlighted the potential of plasma p-tau181 and p-tau217, both of which have shown exceptional sensitivity and specificity to widespread $\mathrm{AD}$ pathology at autopsy and in patients with underlying $\mathrm{AD}$ pathology confirmed by other biomarkers (Janelidze et al., 2020; Karikari et al., 2020; Lantero Rodriguez et al., 2020; Palmqvist et al., 2020; Thijssen et al., 2020). However, due to technical limitations, these results are predominantly based on the Caucasian populations, and studies on blood biomarkers in Chinese populations are hard to achieve and still lacking. To our knowledge, only one study has detected plasma p-tau level in a Chinese population, which mainly focused on the correlation between p-taul81 and cognitive function (Xiao et al., 2021). Further studies are warranted to explore these biomarkers in currently underrepresented populations. Considering the heterogeneity and complexity of AD etiology, it is difficult to use a single biomarker to reflect the comprehensive pathological changes and disease diagnosis. Few domestic studies have integrated plasma $A \beta$, tau, and NfL simultaneously to evaluate their comprehensive diagnostic efficacy for $\mathrm{AD}$ and assess their consistency with classic CSF core biomarkers.

Therefore, in this cross-sectional study, we simultaneously detected the levels of classic biomarkers in the plasma of patients with $\mathrm{AD}$ and cognitively normal $(\mathrm{CN})$ individuals from South China, including $A \beta 42, A \beta 40, t$-tau, and NfL, assessed their performance in discriminating patients with probable $\mathrm{AD}$ from $\mathrm{CN}$ participants to evaluating their ability to diagnose $\mathrm{AD}$.

\section{MATERIALS AND METHODS}

\section{Participants}

A total of 430 individuals, including 277 patients with probable $\mathrm{AD}$ and $153 \mathrm{CN}$ participants, were enrolled from the Department of Neurology, Xiangya Hospital, Central South University, between March 2017 and December 2019. The inclusion criteria for patients were as follows: (1) memory complaints from the patient or guardian; (2) ability to cooperate with physical examination and neuropsychological tests; (3) brain atrophy confirmed by CT or MRI, and (4) diagnosis of probable AD by two or more experienced neurologists from Xiangya Hospital according to the criteria of the National Institute on Aging and Alzheimer's Association (NIA-AA) (McKhann et al., 2011). In this study, the inclusion and exclusion criteria for controls were as follows: (1) no subjective cognitive complaints and no objective impairment in cognitive tests, including the Clinical Dementia Rating Scale $(\mathrm{CDR})(\mathrm{CDR}$ scores $=0)$ and MiniMental State Examination (MMSE; combined with educational attainment, illiteracy $>17$, primary school $>20$, and junior high school and above $>24$ ); (2) no brain organic or functional disease; (3) no hypertension, diabetes, hyperhomocysteinemia, or other systemic diseases; and (4) matched with patients with probable $\mathrm{AD}$ by age and sex.

The study protocol was approved by the Institutional Review Board of Xiangya Hospital of Central South University in China. Written informed consent was obtained from each participant or guardian.

\section{Neuropsychological and Cognitive Assessment}

Participants in the AD group underwent a battery of neuropsychological tests, including the MMSE, Montreal Cognitive Assessment (MoCA), Activities of Daily Living (ADL), and Neuropsychiatric Inventory (NPI). The MMSE was also administered to the $\mathrm{CN}$ group.

Further, all participants were interviewed by two neurologists specializing in neurodegenerative disease, and the severity of cognitive impairment was assessed using the CDR. 


\section{The Apolipoprotein E (APOE) Genotyping}

Venous blood was collected from all participants in tubes containing ethylenediaminetetraacetic acid (EDTA). Genomic DNA was extracted using the standard phenol-chloroform extraction method. All DNA samples were diluted to 50 $\mathrm{ng} / \mu \mathrm{l}$. A 581-bp fragment was amplified using the following primers: forward $5^{\prime}$-CCTACAAATCGGAACTGG-3' and reverse $5^{\prime}$-CTCGAACCAGCTCTTGAG-3'. PCR was performed as previously described (Jiao et al., 2014). Each PCR product was sequenced using an ABI 3730xl DNA analyzer (ABI, Louis, MO, USA).

\section{CSF Collection and Analysis}

The cerebrospinal fluid was obtained from lumbar puncture samples. In this study, as lumbar puncture was carried out on a voluntary basis, a total of 188 patients refused to undergo the procedure, and 89 patients were tested for CSF core biomarkers. Briefly, participants were placed in the left lateral position for lumbar puncture. The L3-L5 intervertebral spaces were selected as the puncture site. The CSF samples were processed within $2 \mathrm{~h}$ after the standard lumbar puncture, and hemorrhagic samples were excluded (Teunissen et al., 2009). Each sample was centrifuged at $2,000 \times g$ for $10 \mathrm{~min}$, and the CSF samples were separated and stored in an enzyme-free microcentrifuge tube at $-80^{\circ} \mathrm{C}$. All samples were subjected to a maximum of two freezethaw cycles. All analyses of CSF core AD biomarkers (A $\beta 42$, $\mathrm{A} \beta 40$, t-tau, and p-tau) were measured using enzyme-linked immunosorbent assay (ELISA) and performed by experienced technicians in strict accordance with the instructions of the manufacturer within 1 week of sample collection. Briefly, samples were added to the reagent wells, and the plate was incubated for $3 \mathrm{~h}$ at $22^{\circ} \mathrm{C} \pm 2^{\circ} \mathrm{C}$. After washing, horseradish peroxidase solution was added and incubated for $90 \mathrm{~min}$ at $22^{\circ} \mathrm{C} \pm 2^{\circ} \mathrm{C}$. The plate was then washed with the provided washing buffer, and substrate solution was added. After $30 \mathrm{~min}$ incubation protected from light, stop-solution was added, and the optical density (OD) was measured using a microplate reader (Thermo, Waltham, MA, USA), at $450 \mathrm{~nm}$, corrected by the reference $\mathrm{OD}$ at $620 \mathrm{~nm}$ within $30 \mathrm{~min}$ of adding the stop solution. Two technical replicates are performed on samples and standards, and the average value of the replicates is used for statistical analysis. All measurements were performed in a blinded manner. Specifically, A $\beta 42$ level in CSF $<651 \mathrm{pg} / \mathrm{ml}$, or $\mathrm{A} \beta 42 / \mathrm{A} \beta 40$ ratio $\leq 0.1$, is defined as positive amyloidosis (corresponding to A+ in the ATN framework); p-tau $>61 \mathrm{pg} / \mathrm{ml}$ in CSF is defined as neurofibrillary tangles (corresponding to $\mathrm{T}+$ in the ATN framework); $\mathrm{t}$-tau $\geq 290 \mathrm{pg} / \mathrm{ml}$ in CSF is defined as nerve cell death (corresponding to $\mathrm{A}+$ in the $\beta$ amyloid deposition, pathologic tau, and neurodegeneration [ATN] framework).

\section{Plasma Protein Quantification}

Venous blood was collected in tubes containing EDTA and centrifuged at $2,000 \times g$ for $10 \mathrm{~min}$ at $4^{\circ} \mathrm{C}$. The obtained plasma was divided into $\sim 500 \mu \mathrm{l}$ aliquots and frozen at $-80^{\circ} \mathrm{C}$. All samples underwent no more than three freezethaw cycles (Keshavan et al., 2018). The samples were rapidly thawed at $22 \pm 2^{\circ} \mathrm{C}$ and then centrifuged at $10,000 \times g$ prior to analysis to prevent any sample debris from interfering with the measurements. Plasma A $\beta 42, \mathrm{~A} \beta 40$, $\mathrm{t}$-tau, and NfL concentrations were measured simultaneously using the singlemolecule array (SIMOA)-HD1 platform (SIMOA; Quanterix, Billerica, MA, USA), which employed an automated SIMOA principle. Briefly, $A \beta 42, A \beta 40$, and t-tau levels were measured using a multiplex array (Neurology 3-Plex A Advantage Kit, N3PA), and NfL levels were measured using a single-analyte array (NF-light). The samples were measured using a twostep immunoassay. All analytical procedures were performed according to the protocol of the manufacturer by well-trained technicians who were blinded to the state of participant and clinical data, according to the protocol of the manufacturer. Samples with coefficients of variance (CV) of $>20 \%$ were excluded from the analyses. In this study, the within-batch CV of all samples was $<5 \%$.

\section{Statistical Analysis}

The normality of the distribution of the variables was assessed. Categorical data were analyzed using the $\chi^{2}$ test. Continuous variables were compared between two independent samples using the $t$-test or the Mann-Whitney $U$ test, and differences between multiple independent samples were compared using the Kruskal-Wallis $\mathrm{H}$ test. Partial correlation analyses were performed to assess the correlations among plasma biomarkers, demographic characteristics, and clinical data. Diagnostic accuracy was evaluated using receiver operating characteristic (ROC) curve analysis and with logistic regression models. The area under the curve (AUC) and representative optimal sensitivity and specificity were used to evaluate the performance of the models. The statistical significance of the difference in AUCs between two different models was analyzed using Delong's test.

All tests were two-tailed, and $p<0.05$ was considered statistically significant. All analyses were performed using SPSS version 24 (IBM, Armonk, NY, USA) and R (version 4.1.0). Data were visualized using Prism 8 software (GraphPad, San Diego, CA, USA).

\section{RESULTS}

\section{Demographic Characteristics}

The demographic characteristics of the patients with probable $\mathrm{AD}$ and $\mathrm{CN}$ participants are summarized in Table 1, including 277 patients with probable $\mathrm{AD}$ and $153 \mathrm{CN}$ participants. The mean age at onset (AAO) was 62.23 years, and the mean disease course was approximately 2.91 years. The sex ratio $(\mathrm{F} / \mathrm{M})$ of patients with probable $\mathrm{AD}$ was $172 / 105$, which matched the CN group $(99 / 54)(p>0.05)$. In parallel, 160 (57.8\%) patients with $\mathrm{AD}$ carried at least one APOE4 allele. There was no significant difference in age at diagnosis (means the age at the time of blood extraction) between patients with probable $\mathrm{AD}$ and $\mathrm{CN}$ participants, but the educational levels were significantly different between the two groups $(p<0.001)$. 
TABLE 1 | Demographic and clinical characteristics of patients with probable AD and healthy controls.

$$
\text { AD }(n=277) \quad \text { Control }(n=153)
$$

p

\begin{tabular}{|c|c|c|c|}
\hline \multicolumn{4}{|c|}{ Demographic and clinical characteristics } \\
\hline $\begin{array}{l}\text { Age at diagnosis (Mean } \\
\pm \mathrm{SD} \text { ) }\end{array}$ & $65.11 \pm 10.57$ & $64.5 \pm 8.2$ & 0.650 \\
\hline $\begin{array}{l}\text { Age at onset (Mean } \pm \\
\text { SD) }\end{array}$ & $62.23 \pm 10.7$ & - & - \\
\hline $\operatorname{Sex}(F / M, n)$ & $172 / 105$ & $99 / 54$ & 0.591 \\
\hline $\begin{array}{l}\text { Education level, years } \\
\text { (Mean } \pm \text { SD) }\end{array}$ & $7.31 \pm 4.38$ & $8.99 \pm 3.52$ & $<0.001^{\star}$ \\
\hline APOE4 $(+/-, n)$ & $160 / 117$ & $26 / 127$ & $<0.001^{*}$ \\
\hline Family history $(+/-, n)$ & $90 / 187$ & - & - \\
\hline $\begin{array}{l}\text { Disease course, years } \\
\text { (Mean } \pm S D)\end{array}$ & $2.91 \pm 2.13$ & - & - \\
\hline MMSE (Mean \pm SD) & $12.0 \pm 6.44$ & $27.7 \pm 2.3$ & $<0.001^{*}$ \\
\hline MoCA (Mean \pm SD) & $7.63 \pm 5.48$ & - & - \\
\hline ADL (Mean \pm SD) & $36.79 \pm 11.78$ & - & - \\
\hline NPI (Mean \pm SD) & $19.20 \pm 14.96$ & - & - \\
\hline \multicolumn{4}{|l|}{$\operatorname{CDR}(n, \%)$} \\
\hline $\mathrm{CDR}=0$ & 0 & $153(100)$ & - \\
\hline $\mathrm{CDR}=1$ & $156(56.3 \%)$ & 0 & - \\
\hline $\mathrm{CDR}=2$ & $108(39.0 \%)$ & 0 & - \\
\hline $\mathrm{CDR}=3$ & $13(4.7 \%)$ & 0 & - \\
\hline \multicolumn{4}{|c|}{ Plasma biomarkers (mean \pm SD) } \\
\hline $\mathrm{A} \beta 42(\mathrm{pg} / \mathrm{ml})$ & $14.18 \pm 3.96$ & $14.58 \pm 3.33$ & $0.02(0.308)$ \\
\hline $\mathrm{A} \beta 40(\mathrm{pg} / \mathrm{ml})$ & $275.77 \pm 63.25$ & $258.48 \pm 50.36$ & $0.002(0.062)$ \\
\hline$A \beta 42 / A \beta 40$ & $0.052 \pm 0.014$ & $0.057 \pm 0.013$ & $<0.001^{\star}(0.056)$ \\
\hline t-tau (pg/ml) & $4.12 \pm 1.25$ & $3.23 \pm 1.12$ & $<0.001^{\star}\left(<0.001^{\#}\right)$ \\
\hline $\mathrm{NfL}(\mathrm{pg} / \mathrm{ml})$ & $28.76 \pm 30.34$ & $14.13 \pm 10.25$ & $<0.001^{*}\left(<0.001^{\#}\right)$ \\
\hline
\end{tabular}

Demographic and clinical characteristics of patients with probable $A D$ and healthy controls. Continuous variable comparisons between two independent samples were conducted via t-test or the Mann-Whitney U test. Categorical data were analyzed using the $\chi^{2}$ test. The $p$-value in the bracket refers to the $p$-value after adjusting for age (means the age at diagnosis), sex, and APOE alleles. The differences of plasma biomarkers between the $A D$ and $C N$ groups were performed correction for multiple comparisons.

${ }^{\star}$ The difference between the groups is statistically significant $(p<0.05)$.

\#The difference between the groups is statistically significant $(p<0.01$, Bonferroni corrected).

$A D$, Alzheimer's disease; $A D L$, Activities of Daily Living; CDR, Clinical Dementia Rating Scale; MMSE, Mini-Mental State Examination; MoCA, Montreal Cognitive Assessment; NPI, Neuropsychiatric Inventory; NfL, neurofilament light chain; $t$-tau, total tau.

\section{Differences in Plasma Biomarkers Levels}

Before correcting for confounding factors, all detected plasma biomarkers, including $A \beta 42, A \beta 40, A \beta 42 / A \beta 40$, t-tau, and NfL, significantly differed between the $\mathrm{AD}$ and $\mathrm{CN}$ groups. Among them, the plasma $A \beta 42$ and $A \beta 42 / 40$ levels were significantly lower in the $\mathrm{AD}$ group than in the $\mathrm{CN}$ group $(p=0.02$ and $p$ $<0.001$, respectively). In parallel, the levels of $A \beta 42 / A \beta 40$, t-tau, and NfL showed an increasing tendency in patients with probable $\mathrm{AD}(p<0.05$; Table 1). However, after adjusting for age, sex, and $A P O E$ alleles, and Bonferroni correction, compared with the $\mathrm{CN}$ group, plasma t-tau, and NfL were significantly increased in the AD group $(p<0.001)$, whereas other plasma biomarkers, including $A \beta 42$ and $A \beta 40$, showed no significant difference
( $p=0.308$ and $p=0.062$, respectively), $\mathrm{A} \beta 42 / \mathrm{A} \beta 40$ showed a decreasing tendency, but the difference was not significant $(p=$ 0.056; Table 1; Figure 1).

\section{Correlations Between Plasma Biomarkers and Demographic Characteristics}

Next, we analyzed the correlations between plasma biomarkers and demographic data, including AAO, age at diagnosis, disease course, and education level. Associations between the levels of plasma biomarkers and demographic data were examined using partial correlation analyses with adjustment for age at diagnosis, sex, and $A P O E$ alleles. In addition, we compared the differences in plasma biomarkers according to sex, family history, and APOE4 alleles distribution (Table 2). After adjustment for age at diagnosis, sex, and $A P O E$ alleles, $A \beta 42, A \beta 40, A \beta 42 / A \beta 40$, and $\mathrm{t}$-tau were not significantly associated with AAO in the probable AD group $(p>0.05)$, but NfL showed a significant association $(r$ $=-0.183, p<0.001)$, which indicated that the earlier the AAO, the higher the level of NfL in plasma. In contrast, there was a significant positive correlation between age at diagnosis and the plasma NfL level $(r=0.235, p<0.001)$. Meanwhile, plasma NfL was positively correlated with the disease course $(r=0.199, p<$ 0.001 ). Moreover, compared with patients with a positive family history of dementia, both plasma $A \beta 42$ and $A \beta 42 / A \beta 40$ were significantly lower in patients without a positive family history of dementia. Regarding APOE alleles, the $\mathrm{A} \beta 42 / \mathrm{A} \beta 40$ ratio, and NfL were significantly lower in patients carrying the APOE4 alleles than in non-carriers ( $p=0.033$ and $p=0.034$, respectively), whereas there were no significant differences in other plasma biomarkers between the two subgroups (Table 2).

\section{Correlations Between Plasma Biomarker Levels and Neuropsychological Assessments}

We analyzed the correlations between plasma biomarkers and neuropsychological assessments, including MMSE, MoCA, ADL, and NPI (Table 2). Associations between the levels of plasma biomarkers and neuropsychological assessments were examined using partial correlation analyses with adjustment for age, sex, $A P O E$ alleles, and education level. The results showed that all the plasma biomarkers showed no significant association with neuropsychological assessments, including MMSE, MoCA, ADL, and NPI.

To further analyze the correlations between plasma biomarkers levels and disease severity, the patients with probable $\mathrm{AD}$ were divided into three subgroups according to the CDR score. No significant difference in plasma biomarkers was found among patients with different disease severity.

\section{Correlations Between Plasma and CSF Biomarkers}

According to the ATN diagnosis framework, not all clinically diagnosed patients with probable $\mathrm{AD}$ were compatible with the biologically defined AD. Specifically, among 89 patients who underwent lumbar puncture, 70 (78.7\%) patients were diagnosed with $\mathrm{AD}$ continuum $(\mathrm{A}+\mathrm{T}+\mathrm{N}+$ : 39 cases, $\mathrm{A}+\mathrm{T}+\mathrm{N}-$ : 


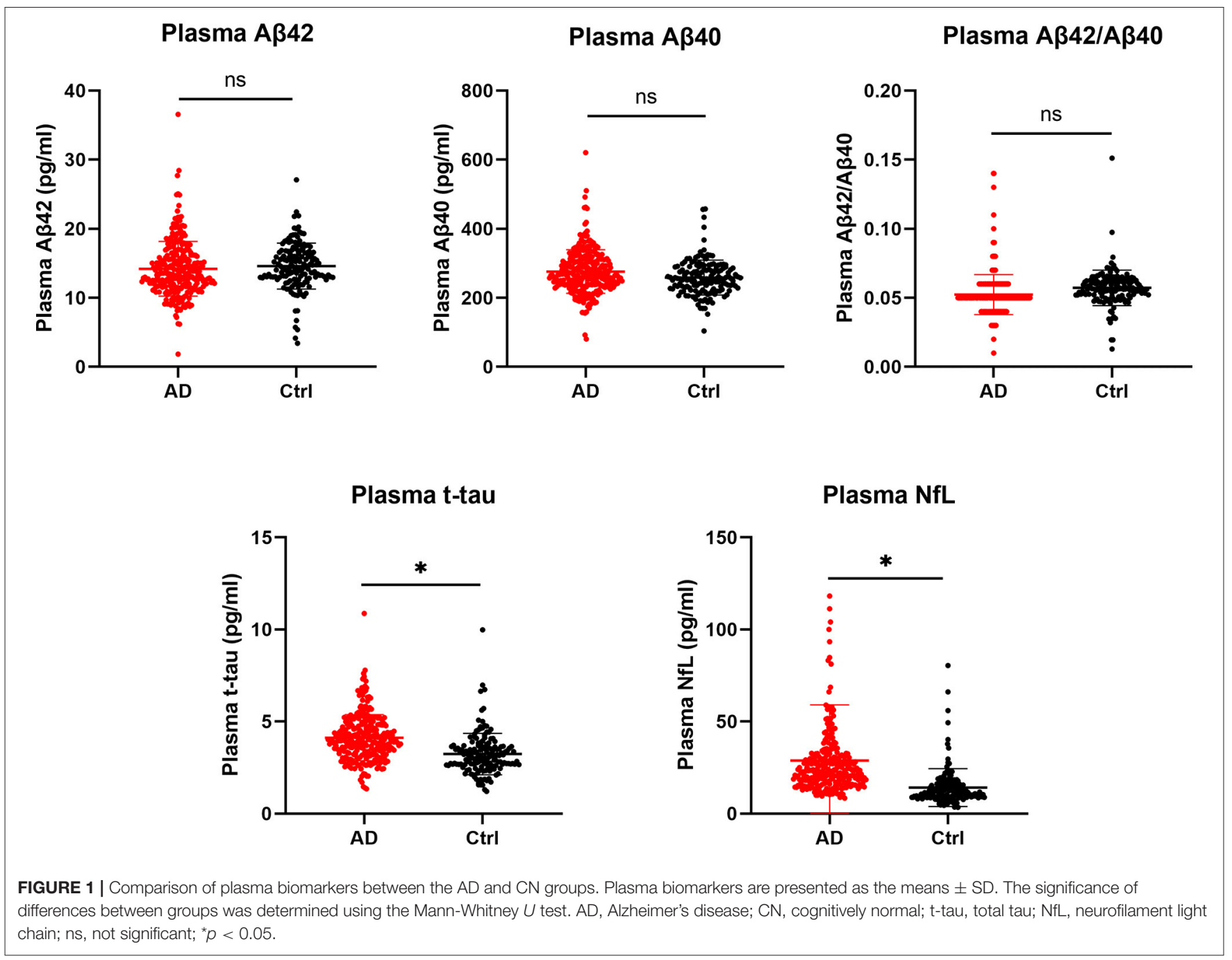

8 cases, $\mathrm{A}+\mathrm{T}-\mathrm{N}+: 9$ cases, $\mathrm{A}+\mathrm{T}-\mathrm{N}-$ : 14 cases). The correlations between plasma and CSF biomarker levels in patients with probable $\mathrm{AD}$ were determined to assess the efficacy of plasma biomarkers in reflecting changes in brain pathology, as shown in Table 3. After adjusting for age, sex, and APOE alleles, only plasma t-tau was positively correlated with $\mathrm{t}$-tau in the CSF $(r=319, p=0.003)$.

\section{Diagnostic Performance of Plasma Biomarkers}

Finally, ROC curves were generated to evaluate the performance of the plasma biomarkers to discriminate patients with probable $\mathrm{AD}$ from controls (Figure 2). The cutoff value and its corresponding sensitivity and specificity were calculated using the maximum Youden index (Table 4). As a single plasma biomarker, NfL displayed the best diagnostic efficacy (AUC = 0.85 , sensitivity $=73.28 \%$, specificity $=83.00 \%$ ). In addition, based on the results of differences in plasma biomarker levels, the model combining plasma t-tau and NfL showed the best diagnostic performance (AUC $=0.86$, sensitivity $=83.75 \%$, specificity $=76.47 \%)$. Furthermore, when age, sex, and APOE alleles were included in the combined model, it showed the best performance to distinguish probable $\mathrm{AD}$ from $\mathrm{CN}$ participants $($ AUC $=0.89$, sensitivity $=82.31 \%$, specificity $=83.66 \%$, which was significantly better than other models $(p<0.05)$. In addition, we extracted 89 patients with AD who underwent CSF biomarker detection and analyzed the diagnostic performance of their plasma biomarkers through the ROC curve. The results showed that when plasma t-tau and NfL are included in the model, the diagnostic efficiency is better than that of any single plasma biomarker. After incorporating age, sex, and $A P O E$ alleles into the model, there was no statistical difference in the diagnostic performance of the two models, but the AUC of the latter reached the best $(\mathrm{AUC}=0.89$, sensitivity $=78.65 \%$, specificity $=88.88 \%$; Table 4).

\section{DISCUSSION}

This study aimed to evaluate the ability of plasma biomarkers, including $\mathrm{A} \beta$, t-tau, and $\mathrm{NfL}$, to detect probable $\mathrm{AD}$ in a Chinese population. We first determined the differences in plasma biomarkers between patients with probable $\mathrm{AD}$ 
TABLE 2 | Correlations of plasma biomarkers with demographics and neuropsychological assessments in patients with AD.

\begin{tabular}{|c|c|c|c|c|c|c|c|c|c|c|}
\hline \multirow[t]{2}{*}{ CSF biomarkers } & \multicolumn{2}{|c|}{ Plasma $A \beta 42$} & \multicolumn{2}{|c|}{ Plasma $A \beta 40$} & \multicolumn{2}{|c|}{ Plasma A $\beta 42 / A \beta 40$} & \multicolumn{2}{|c|}{ Plasma t-tau } & \multicolumn{2}{|c|}{ Plasma NfL } \\
\hline & $r$ & $p$ & $r$ & $p$ & $\boldsymbol{R}$ & $p$ & $r$ & $p$ & $r$ & $p$ \\
\hline Age at onset & -0.041 & 0.503 & -0.018 & 0.763 & -0.007 & 0.907 & -0.025 & 0.682 & -0.198 & $<0.001^{*}$ \\
\hline Age at diagnosis & 0.040 & 0.513 & 0.081 & 0.184 & -0.048 & 0.426 & 0.011 & 0.861 & 0.235 & $<0.001^{*}$ \\
\hline $\operatorname{Sex}(F / M)$ & - & 0.285 & - & 0.097 & - & 0.619 & - & 0.425 & - & 0.077 \\
\hline Education level (years) & -0.024 & 0.691 & -0.039 & 0.518 & 0.025 & 0.684 & 0.058 & 0.336 & 0.105 & 0.083 \\
\hline Disease course (years) & 0.041 & 0.497 & 0.018 & 0.765 & 0.007 & 0.912 & 0.021 & 0.729 & 0.199 & $<0.001^{\star}$ \\
\hline Family history (+/-) & - & $<0.001^{\star}$ & - & 0.627 & - & $<0.001^{\star}$ & - & 0.630 & - & 0.218 \\
\hline APOE4 (+/-) & - & 0.349 & - & 0.465 & - & $0.033^{*}$ & - & 0.666 & - & $0.034^{\star}$ \\
\hline MMSE & 0.054 & 0.375 & 0.078 & 0.198 & -0.023 & 0.701 & -0.079 & 0.191 & -0.044 & 0.474 \\
\hline MoCA & 0.069 & 0.258 & 0.085 & 0.160 & -0.015 & 0.811 & -0.059 & 0.334 & 0.008 & 0.891 \\
\hline ADL & -0.115 & 0.058 & -0.090 & 0.139 & -0.006 & 0.920 & -0.014 & 0.818 & -0.035 & 0.566 \\
\hline NPI & -0.051 & 0.402 & 0.021 & 0.731 & -0.071 & 0.242 & 0.012 & 0.845 & 0.021 & 0.729 \\
\hline
\end{tabular}

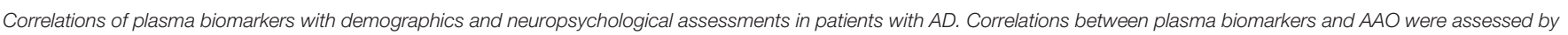

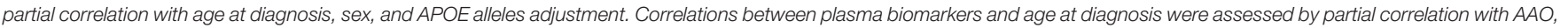

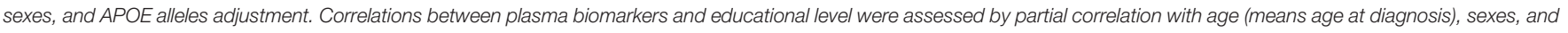

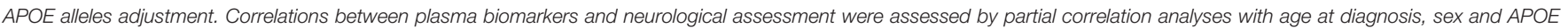
alleles, educational levels adjustment.

*The difference between the groups is statistically significant $(p<0.05)$.

AD, Alzheimer's disease; ADL, Activities of Daily Living; MMSE, Mini-Mental State Examination; MoCA, Montreal Cognitive Assessment; NPI, Neuropsychiatric Inventory; NfL, neurofilament light chain; $t$-tau, total tau.

TABLE 3 | Correlations between plasma biomarkers and CSF biomarkers in patients with $\operatorname{AD}(n=89)$.

\begin{tabular}{|c|c|c|c|c|c|c|c|c|c|c|}
\hline \multirow[t]{2}{*}{ CSF biomarkers } & \multicolumn{2}{|c|}{ Plasma $A \beta 42$} & \multicolumn{2}{|c|}{ Plasma $A \beta 40$} & \multicolumn{2}{|c|}{ Plasma A $\beta 42 / A \beta 40$} & \multicolumn{2}{|c|}{ Plasma t-tau } & \multicolumn{2}{|c|}{ Plasma NfL } \\
\hline & $r$ & $p$ & $r$ & $p$ & $r$ & $p$ & $\boldsymbol{R}$ & $p$ & $r$ & $p$ \\
\hline$A \beta 42$ & 0.081 & 0.461 & 0.098 & 0.370 & -0.011 & 0.923 & -0.061 & 0.579 & 0.017 & 0.875 \\
\hline$A \beta 40$ & -0.081 & 0.458 & -0.090 & 0.410 & 0.024 & 0.823 & -0.158 & 0.146 & -0.013 & 0.907 \\
\hline$A \beta 42 / A \beta 40$ & 0.166 & 0.126 & 0.209 & 0.053 & 0.037 & 0.738 & -0.081 & 0.456 & 0.180 & 0.097 \\
\hline p-tau & -0.150 & 0.169 & -0.166 & 0.128 & -0.002 & 0.982 & 0.065 & 0.551 & -0.141 & 0.197 \\
\hline t-tau & -0.115 & 0.293 & -0.151 & 0.164 & -0.019 & 0.862 & 0.319 & $0.003^{*}$ & -0.121 & 0.268 \\
\hline
\end{tabular}

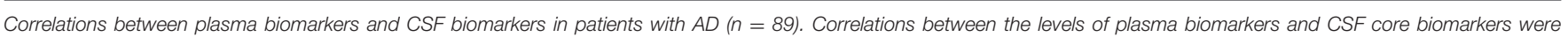
assessed with partial correlation analyses with age, sexes, and APOE alleles adjustment. Age here refers to the age at diagnosis.

${ }^{\star}$ The difference of the correlations between these biomarkers is statistically significant $(p<0.05)$.

$A D$, Alzheimer's disease; NfL, neurofilament light chain; t-tau, total tau.

and CN participants. Second, a comprehensive model with high diagnostic efficacy of $\mathrm{AD}$ was constructed, including plasma t-tau, NfL, age, sex, and APOE alleles, which can be applied to perform preliminary screening in populations with a high risk of $\mathrm{AD}$ and may effectively reduce the application of lumbar puncture and PET examinations in clinical practice.

At present, a lot of efforts are devoted to comparing the difference of plasma $\mathrm{A} \beta$ between patients with $\mathrm{AD}$ and control, trying to diagnose $\mathrm{AD}$ through the less invasive and easily acceptable method. However, the results of different studies are still controversial. Studies adopting the SIMOA platform showed lower levels of plasma $A \beta 42$ and $A \beta 42 / A \beta 40$ in patients with AD than in controls (Janelidze et al., 2016; Li et al., 2019; Tosun et al., 2021). By contrast, some studies found that the levels of $\mathrm{A} \beta 42$ and $A \beta 42 / \mathrm{A} \beta 40$ were significantly increased in patients with mild cognitive impairment (MCI) or AD (Teunissen et al.,
2018; Palmqvist et al., 2019). However, several studies showed that there were no significant differences in the plasma $A \beta$ levels between patients with $\mathrm{AD}$ and controls (Hsu et al., 2017; Lövheim et al., 2017). The low abundance of $A \beta$ in plasma and detection methods with different sensitivities, such as ELISA, SIMOA, immunomagnetic reduction (IMR), and Elecsys immunoassays, are the main reasons for these conflicting findings $(\mathrm{Qu}$ et al., 2021). To our knowledge, few domestic studies have evaluated these plasma biomarkers simultaneously in large cohorts from China or analyzed their correlations with typical AD biomarkers (Lue et al., 2017; Shi et al., 2019). In the present study, after adjusting for age, sex, and $A P O E$ alleles, the level of plasma $\mathrm{A} \beta 42 / \mathrm{A} \beta 40$ showed a decreasing tendency but not a significant difference in patients with probable $\mathrm{AD}$; meanwhile, the individual levels of plasma $A \beta 42$ and $A \beta 40$ were not significantly different from those of controls, which was consistent with the previous study (Olsson et al., 2016). A $\beta 40$ is always used 
as a reference peptide to potentially explain the difference in CSF concentrations between individuals and the difference in sample preanalytical processing (Schauer et al., 2018). As for the correlation between plasma $\mathrm{A} \beta$ and neuropsychological assessment, previous studies have reported the association between plasma $A \beta$ levels and cognitive performance (Hanon et al., 2018; Chen et al., 2019), which indicates that plasma $A \beta$ can potentially reflect the cognitive function even monitor the progression of $\mathrm{AD}$. In this study, after adjusting for confounding factors, there was no significant correlation between plasma $A \beta$ and neuropsychological assessment.

Approximately $30-50 \%$ of blood $\mathrm{A} \beta$ is derived from a brainto-blood transport mechanism, and a dynamic equilibrium exists between the peripheral blood and the CNS (Roberts et al., 2014). The levels of $A \beta$ in the plasma reflect pathological changes in the brain to some extent. Robust studies have shown that plasma $\mathrm{A} \beta$ levels can reflect $\mathrm{A} \beta$ pathology in the brain using amyloid PET or CSF A $\beta$ levels as the positive reference (Park et al., 2017; Hanon et al., 2018; Pérez-Grijalba et al., 2019; Schindler et al., 2019). Furthermore, the ratio in plasma appears to be

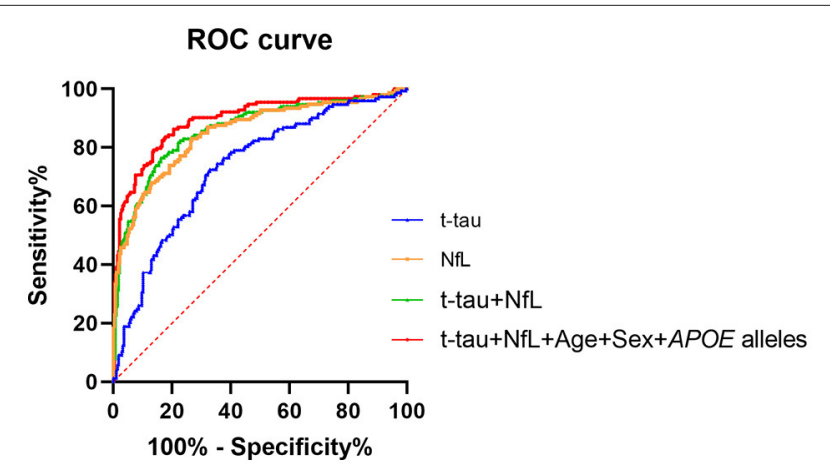

FIGURE 2 | ROC curve analysis of plasma biomarkers for the AD diagnosis. NfL levels in plasma exhibited the best diagnostic efficacy as a single indicator (AUC $=0.85$, sensitivity $=73.28 \% \%$, specificity $=83.00 \%$ ). Combined with $\mathrm{t}$-tau and NfL levels in plasma, the diagnostic performance was significantly improved ( $A \cup C=0.86$, sensitivity $=83.75 \%$, specificity $=76.47 \%$ ). The diagnostic model combining plasma t-tau and NfL levels, age, sex, and APOE alleles showed the best performance for the identification of probable AD (AUC $=0.89$, sensitivity $=82.31 \%$, specificity $=83.66 \%$ ). Delong test was performed to compare the difference of different diagnostic models. AUC, area under the curve; ROC, receiver operating characteristic curve; NfL, neurofilament light chain; t-tau, total tau. associated with the increased risk of progression to AD dementia (Verberk et al., 2018). However, in this study, the associations of plasma and CSF A $\beta$ were not significant. There are several possibilities to explain these results. First, in the present study, only 89 patients with probable AD were tested for CSF core biomarkers, and the small sample size of CSF cannot reflect the true relationship of $A \beta$ between plasma and CSF. Second, in this study, the inclusion criteria of patients with $\mathrm{AD}$ were the NIAAA criteria and not all patients met the biological definition of $\mathrm{AD}$. Considering that the clinical symptoms of different types of neurodegenerative dementia have a high degree of overlap, there are several clinically diagnosed patients with probable AD without Alzheimer's pathology. Third, the different detection methods used for markers in CSF and plasma may also be one of the reasons for the weak correlation. Finally, in this study, the different disease severity of patients with $\mathrm{AD}$ may also explain part of the results.

In the present study, plasma t-tau levels were significantly higher in patients with probable $\mathrm{AD}$ than in healthy controls, similar to the findings of most published results (Mattsson et al., 2016; Lue et al., 2017), whereas several studies also found that plasma t-tau levels showed the opposite result or no significant difference between them (Sparks et al., 2012; Verberk et al., 2018; $\mathrm{Qu}$ et al., 2021). In a large meta-analysis, it was confirmed that patients with $\mathrm{AD}$ had higher plasma t-tau levels than controls, which may reflect neuronal damage as a nonspecific marker (Jack et al., 2018; Ding et al., 2021). Mattson et al. found that the higher plasma t-tau level was associated with $\mathrm{AD}$ dementia and showed significant correlations with poor cognition, greater atrophy, and hypometabolism during follow-up in the $\mathrm{AD}$ Neuroimaging Initiative study (Mattsson et al., 2016). In our study, after adjusting for age, sex, and $A P O E$ alleles, plasma ttau was weakly correlated with CSF t-tau, which is consistent with previous studies that the association between plasma $\mathrm{t}$ tau with CSF t-tau was weak or nonsignificant (Müller et al., 2017; Pase et al., 2019). Notably, accumulating evidence supports that plasma $\mathrm{p}$-tau, the most promising biomarker for $\mathrm{AD}$, shows outstanding performance in differential diagnosis, in relation to other biomarkers, neuropathology, prediction, progression monitoring, and prognosis (Mielke et al., 2018; Janelidze et al., 2020, 2021; Palmqvist et al., 2020). Due to the low concentration in the samples and limitations associated with the methodology, we did not measure p-tau levels in plasma. In our next study, we will increase the sample size to reevaluate whether plasma t-tau

TABLE 4 | Performance of plasma biomarkers for probable AD diagnosis.

\begin{tabular}{|c|c|c|c|c|c|}
\hline Plasma biomarkers & Sensitivity (\%) & Specificity (\%) & AUC & $95 \% \mathrm{Cl}$ & $p$ \\
\hline t-tau & $67.14(66.29)$ & $72.54(78.43)$ & $0.73(0.74)$ & $0.68-0.78(0.67-0.80)$ & $<0.001^{\star}\left(<0.001^{*}\right)$ \\
\hline NfL & $73.28(77.52)$ & $83.00(82.35)$ & $0.85(0.86)$ & $0.81-0.89(0.78-0.90)$ & $0.002^{*}(0.08)$ \\
\hline $\mathrm{t}$-tau $+\mathrm{NfL}+$ age + sex $+A P O E$ alleles & $82.31(78.65)$ & $83.66(88.88)$ & $0.89(0.89)$ & $0.86-0.92(0.86-0.94)$ & \\
\hline
\end{tabular}

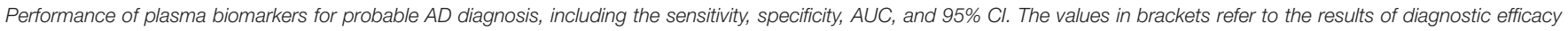

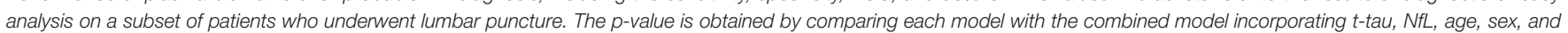

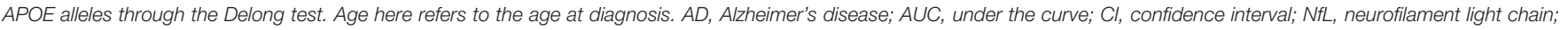
$t$-tau, total tau. ${ }^{*}$ The difference of the correlations between these biomarkers is statistically significant $(p<0.05)$. 
levels predict cognitive function and $\mathrm{AD}$ pathology and assess the utility of plasma $\mathrm{p}$-tau levels for discriminating patients with $\mathrm{AD}$ from controls in the Chinese population.

Neurofilament light chain is a sensitive and promising biomarker for neurodegenerative disease, as it is released into CSF and plasma after axonal damage (Kuhle et al., 2015; Weston et al., 2017). Recently, Quiroz et al. found that plasma NfL levels increased with age and began to differentiate in PSEN1 E280A mutation carriers from noncarriers as early as 22 years of age based on a large kindred study of patients with $\mathrm{AD}$ (Quiroz et al., 2020). Consistent with these results, the plasma NfL concentration was correlated with age and increased significantly as individuals aged in the present study. Meanwhile, the association between plasma NfL levels was positively correlated with disease course, which supports the hypothesis that the NfL is a sensitive marker of progressive myelinated axonal damage in the early stage of $\mathrm{AD}$. However, it is not a specific indicator of the typical pathological changes associated with $\mathrm{AD}$ (Disanto et al., 2017). The correlation analysis showed that plasma NfL levels were not associated with AD core biomarkers in CSF, which is inconsistent with some previous study findings (Lewczuk et al., 2018; Mattsson et al., 2019). Combined with a recent longitudinal study showing that the plasma NfL level of participants who developed $\mathrm{AD}$ increased at a rate that was consistently higher than that of $\mathrm{CN}$ participants, and as early as 10 years before the clinical diagnosis of AD (de Wolf et al., 2020), these data indicate that it maybe a stable and useful biomarker for disease identification and for monitoring disease progression.

Notably, the results suggested that these plasma indicators were promising for discriminating patients with probable $\mathrm{AD}$ from $\mathrm{CN}$ participants. The peripheral biomarker panel as an initial screening strategy to identify people who should undergo further examinations, such as PET imaging or CSF testing, might be a critical step forward. Additionally, given the low concentrations of markers in plasma and the limited detection sensitivity, methodological limitations still exist. In this study, we unified the detection method by SIMOA technology to minimize possible experimental errors and promote the accurate detection of plasma biomarkers.

There are some limitations to this study. First, the study was a cross-sectional study without longitudinal follow-up to determine the differences in the trajectories of plasma biomarkers of patients in different $\mathrm{AD}$ stages and $\mathrm{CN}$ individuals. Second, due to the invasive characteristics of lumbar puncture, we failed to obtain the CSF data of all the participants in this study. Importantly, as the inclusion criteria for patients were probable $\mathrm{AD}$ but not biologically proven $\mathrm{AD}$, there maybe several "AD" participants without Alzheimer's pathology as the primary cause of their symptoms, or controls with Alzheimer's pathology since $\mathrm{AD}$ is a continuum, where participants may present without any symptoms. Finally, patients with non-AD dementia were not recruited to compare differences in plasma biomarkers and verify the ability of the panel to distinguish patients with $\mathrm{AD}$ dementia from patients with other forms of dementia. Thus, head-to-head comparisons based on large-scale prospective studies that adopt unified and standardized inclusion criteria, detection, and analysis methods are necessary to assess the ability of plasma biomarkers for $\mathrm{AD}$ diagnosis in a Chinese population and their utility in monitoring pathological changes in the brain.

In summary, plasma biomarkers, including t-tau and NfL levels, were significantly different between the $\mathrm{AD}$ and $\mathrm{CN}$ groups after adjusting for age, sex, and $A P O E$ alleles. The diagnostic model that included t-tau, NfL levels, age, sex, and APOE alleles showed the best performance in discriminating patients with probable $\mathrm{AD}$ from $\mathrm{CN}$ participants. Although the accurate diagnosis of $\mathrm{AD}$ using plasma biomarkers is still challenging, the combination of multiple blood biomarkers can identify patients with probable $\mathrm{AD}$ from controls and is expected to serve as a potential method for pre-screening probable $\mathrm{AD}$.

\section{DATA AVAILABILITY STATEMENT}

The raw data supporting the conclusions of this article will be made available by the authors, without undue reservation.

\section{ETHICS STATEMENT}

The studies involving human participants were reviewed and approved by the Ethics Committee of Xiangya Hospital of the Central South University. The patients/participants provided their written informed consent to participate in this study.

\section{AUTHOR CONTRIBUTIONS}

BJ, HL, and LS: study design, acquisition of data, analysis and interpretation of data, and drafting/revising the manuscript. LG, $\mathrm{XL}$, and YZho: analyzed the data and revised the manuscript for intellectual content. LW, XX, LuZ, XW, YJ, QY, YZhu, WZ, LiZ, JW, XY, and BT: data collection and analysis. All authors contributed to the article and approved the submitted version.

\section{FUNDING}

This study was supported by the National Key R\&D Program of China (Nos. 2020YFC2008500, 2017YFC0840100, and 2017 YFC0840104 to LS, and No. 2018YFC1312003 to JW), the National Natural Science Foundation of China (Nos. 81671075 and 81971029 to LS, No. 82071216 to BJ, and No. 81901171 to XL), Hunan Innovative Province Construction Project (No. 2019SK2335 to BT), and the Youth Science Foundation of Xiangya Hospital (No. 2018Q020 to XL).

\section{ACKNOWLEDGMENTS}

The authors thank all the patients and their families, and all healthy volunteers for their involvement in this study. The authors are grateful to all our colleagues in the Department of Neurology, Xiangya Hospital, Central South University, China. 


\section{REFERENCES}

Bridel, C., van Wieringen, W. N., Zetterberg, H., Tijms, B. M., Teunissen, C. E., Alvarez-Cermeño, J. C., et al. (2019). Diagnostic value of cerebrospinal fluid neurofilament light protein in neurology. JAMA Neurol. 76:1035. doi: 10.1001/jamaneurol.2019.1534

Chen, T., Lee, Y., Lin, S., Chen, J., Hu, C., Wang, P., et al. (2019). Plasma aß42 and total tau predict cognitive decline in amnestic mild cognitive impairment. Sci. Rep. UK 9, 1-10. doi: 10.1038/s41598-019-50315-9

Christina, P. (2018). World Alzheimer Report 2018: The State of the Art of Dementia Research: New Frontiers. London: Alzheimer's Disease International.

de Wolf, F., Ghanbari, M., Licher, S., McRae-McKee, K., Gras, L., Weverling, G. J., et al. (2020). Plasma tau, neurofilament light chain and amyloid- $\beta$ levels and risk of dementia; a population-based cohort study. Brain 143, 1220-1232. doi: 10.1093/brain/awaa054

Desikan, R. S., Cabral, H. J., Hess, C. P., Dillon, W. P., Glastonbury, C. M., Weiner, M. W., et al. (2009). Automated mri measures identify individuals with mild cognitive impairment and Alzheimer's disease. Brain 132, 2048-2057. doi: 10.1093/brain/awp123

Ding, X., Zhang, S., Jiang, L., Wang, L., Li, T., and Lei, P. (2021). Ultrasensitive assays for detection of plasma tau and phosphorylated tau 181 in Alzheimer's disease: a systematic review and meta-analysis. Transl. Neurodegener. 10, 1-14. doi: 10.1186/s40035-021-00234-5

Disanto, G., Barro, C., Benkert, P., Naegelin, Y., Schädelin, S., Giardiello, A., et al. (2017). Serum neurofilament light: a biomarker of neuronal damage in multiple sclerosis. Ann. Neurol. 81, 857-870. doi: 10.1002/ana.24954

Hanon, O., Vidal, J., Lehmann, S., Bombois, S., Allinquant, B., Tréluyer, J., et al. (2018). Plasma amyloid levels within the Alzheimer's process and correlations with central biomarkers. Alzheimers Dement. 14, 858-868. doi: 10.1016/j.jalz.2018.01.004

Hsu, J., Lee, W., Liao, Y., Wang, S., and Fuh, J. (2017). The clinical significance of plasma clusterin and $\mathrm{a} \beta$ in the longitudinal follow-up of patients with Alzheimer's disease. Alzheimers Res. Ther. 9, 1-11. doi: $10.1186 / \mathrm{s} 13195-017-0319-\mathrm{x}$

Jack, C. R., Bennett, D. A., Blennow, K., Carrillo, M. C., Dunn, B., Haeberlein, S. B., et al. (2018). NIA-AA research framework: toward a biological definition of Alzheimer's disease. Alzheimers Dement. 14, 535-562. doi: 10.1016/j.jalz.2018.02.018

Jack, C. R. P. D., Knopman, D. S. P., Jagust, W. J. P., Petersen, R. C. P., Weiner, M. W. P., Aisen, P. S. P., et al. (2013). Tracking pathophysiological processes in Alzheimer's disease: an updated hypothetical model of dynamic biomarkers. Lancet Neurol. 12, 207-216. doi: 10.1016/S1474-4422(12)70291-0

Janelidze, S., Berron, D., Smith, R., Strandberg, O., Proctor, N. K., Dage, J. L., et al. (2021). Associations of plasma phospho-tau217 levels with tau positron emission tomography in early Alzheimer disease. JAMA Neurol. 78, 149-156. doi: 10.1001/jamaneurol.2020.4201

Janelidze, S., Mattsson, N., Palmqvist, S., Smith, R., Beach, T. G., Serrano, G. E., et al. (2020). Plasma p-tau181 in Alzheimer's disease: relationship to other biomarkers, differential diagnosis, neuropathology and longitudinal progression to Alzheimer's dementia. Nat. Med. 26, 379-386. doi: 10.1038/s41591-020-0755-1

Janelidze, S., Stomrud, E., Palmqvist, S., Zetterberg, H., van Westen, D., Jeromin, A., et al. (2016). Plasma beta-amyloid in Alzheimer's disease and vascular disease. Sci. Rep. 6:26801. doi: 10.1038/srep26801

Jansen, W. J., Ossenkoppele, R., Knol, D. L., Tijms, B. M., Scheltens, P., Verhey, F. R. J., et al. (2015). Prevalence of cerebral amyloid pathology in persons without dementia. JAMA 313:1924. doi: 10.1001/jama.2015.4668

Jiao, B., Liu, X., Tang, B., Hou, L., Zhou, L., Zhang, F., et al. (2014). Investigation of trem2, pld3, and unc $5 \mathrm{c}$ variants in patients with Alzheimer's disease from mainland china. Neurobiol. Aging 35, 2422-2429. doi: 10.1016/j.neurobiolaging.2014.04.025

Karikari, T. K., Pascoal, T. A., Ashton, N. J., Janelidze, S., Benedet, A. L., Rodriguez, J. L., et al. (2020). Blood phosphorylated tau 181 as a biomarker for Alzheimer's disease: a diagnostic performance and prediction modelling study using data from four prospective cohorts. Lancet Neurol. 19, 422-433. doi: 10.1016/S1474-4422(20)30071-5

Keshavan, A., Heslegrave, A., Zetterberg, H., and Schott, J. M. (2018). Stability of blood-based biomarkers of Alzheimer's disease over multiple freeze-thaw cycles. Alzheimers Dement (Amst) 10, 448-451. doi: 10.1016/j.dadm.2018.06.001

Kuhle, J., Gaiottino, J., Leppert, D., Petzold, A., Bestwick, J. P., Malaspina, A., et al. (2015). Serum neurofilament light chain is a biomarker of human spinal cord injury severity and outcome. J. Neurol. Neurosurg. Psychiatry 86, 273-279. doi: 10.1136/jnnp-2013-307454

Landau, S. M., Mintun, M. A., Joshi, A. D., Koeppe, R. A., Petersen, R. C., Aisen, P. S., et al. (2012). Amyloid deposition, hypometabolism, and longitudinal cognitive decline. Ann. Neurol. 72, 578-586. doi: 10.1002/ana.23650

Lantero Rodriguez, J., Karikari, T. K., Suárez-Calvet, M., Troakes, C., King, A., Emersic, A., et al. (2020). Plasma p-tau181 accurately predicts Alzheimer's disease pathology at least 8 years prior to post-mortem and improves the clinical characterisation of cognitive decline. Acta Neuropathol. 140, 267-278. doi: 10.1007/s00401-020-02195-x

Lewczuk, P., Ermann, N., Andreasson, U., Schultheis, C., Podhorna, J., Spitzer, P., et al. (2018). Plasma neurofilament light as a potential biomarker of neurodegeneration in Alzheimer's disease. Alzheimers Res. Ther. 10:71. doi: 10.1186/s13195-018-0404-9

Li, W., Shen, Y., Tian, D., Bu, X., Zeng, F., Liu, Y., et al. (2019). Brain amyloid$\beta$ deposition and blood biomarkers in patients with clinically diagnosed Alzheimer's disease. J. Alzheimers Dis. 69, 169-178. doi: 10.3233/JAD-190056

Lövheim, H., Elgh, F., Johansson, A., Zetterberg, H., Blennow, K., and Hallmans, G., et al. (2017). Plasma concentrations of free amyloid $\beta$ cannot predict the development of Alzheimer's disease. Alzheimers Dement. 13, 778-782. doi: 10.1016/j.jalz.2016.12.004

Lue, L., Sabbagh, M. N., Chiu, M., Jing, N., Snyder, N. L., Schmitz, C., et al. (2017). Plasma levels of $\mathrm{a} \beta 42$ and tau identified probable Alzheimer's dementia: findings in two cohorts. Front. Aging Neurosci. 9:226. doi: 10.3389/fnagi.2017.00226

Mattsson, N., Andreasson, U., Zetterberg, H., and Blennow, K. (2017). Association of plasma neurofilament light with neurodegeneration in patients with Alzheimer disease. JAMA Neurol. 74, 557-566. doi: 10.1001/jamaneurol.2016.6117

Mattsson, N., Cullen, N. C., Andreasson, U., Zetterberg, H., and Blennow, K. (2019). Association between longitudinal plasma neurofilament light and neurodegeneration in patients with Alzheimer disease. JAMA Neurol. 76, 791-799. doi: 10.1001/jamaneurol.2019.0765

Mattsson, N., Zetterberg, H., Hansson, O., Andreasen, N., Parnetti, L., Jonsson, M., et al. (2009). Csf biomarkers and incipient Alzheimer disease in patients with mild cognitive impairment. JAMA 302, 385-393. doi: 10.1001/jama.2009.1064

Mattsson, N., Zetterberg, H., Janelidze, S., Insel, P. S., Andreasson, U., Stomrud, E., et al. (2016). Plasma tau in Alzheimer disease. Neurology 87, 1827-1835. doi: 10.1212 /WNL.0000000000003246

Mayeli, M., Mirshahvalad, S. M., Aghamollaii, V., Tafakhori, A., Abdolalizadeh, A., Rahmani, F., et al. (2019). Plasma neurofilament light chain levels are associated with cortical hypometabolism in Alzheimer disease signature regions. J. Neuropathol. Exp. Neurol. 78, 70-716. doi: 10.1093/jnen/nlz054

McKhann, G. M., Knopman, D. S., Chertkow, H., Hyman, B. T., Jack, C. R., Kawas, C. H., et al. (2011). The diagnosis of dementia due to Alzheimer's disease: recommendations from the national institute on aging-Alzheimer's association workgroups on diagnostic guidelines for Alzheimer's disease. Alzheimers Dement. 7, 263-269. doi: 10.1016/j.jalz.2011.03.005

Mielke, M. M., Hagen, C. E., Wennberg, A. M. V., Airey, D. C., Savica, R., Knopman, D. S., et al. (2017). Association of plasma total tau level with cognitive decline and risk of mild cognitive impairment or dementia in the mayo clinic study on aging. JAMA Neurol. 74:1073. doi: 10.1001/jamaneurol.2017.1359

Mielke, M. M., Hagen, C. E., Xu, J., Chai, X., Vemuri, P., Lowe, V. J., et al. (2018), Plasma phospho-tau 181 increases with Alzheimer's disease clinical severity and is associated with tau- and amyloid-positron emission tomography. Alzheimer's Dement. 14, 989-997. doi: 10.1016/j.jalz.2018.02.013

Müller, S., Preische, O., Göpfert, J. C., Yañez, V. A. C., Joos, T. O., Boecker, H., et al. (2017). Tau plasma levels in subjective cognitive decline: results from the delcode study. Sci. Rep. 7, 1-6. doi: 10.1038/s41598-017-08779-0

Olsson, B., Lautner, R., Andreasson, U., Öhrfelt, A., Portelius, E., Bjerke, M., et al. (2016). Csf and blood biomarkers for the diagnosis of Alzheimer's disease: a systematic review and meta-analysis. Lancet Neurol. 15, 673-684. doi: $10.1016 / S 1474-4422(16) 00070-3$ 
Palmqvist, S., Janelidze, S., Quiroz, Y. T., Zetterberg, H., Lopera, F., Stomrud, E., et al. (2020). Discriminative accuracy of plasma phospho-tau217 for Alzheimer disease vs. other neurodegenerative disorders. JAMA 324, 772-781. doi: 10.1001/jama.2020.12134

Palmqvist, S., Janelidze, S., Stomrud, E., Zetterberg, H., Karl, J., Zink, K., et al. (2019). Performance of fully automated plasma assays as screening tests for Alzheimer disease-related $\beta$-amyloid status. JAMA Neurol. 76:1060. doi: 10.1001/jamaneurol.2019.1632

Park, J., Han, S., Cho, H. J., Byun, M. S., Yi, D., Choe, Y. M., et al. (2017). Chemically treated plasma a $\beta$ is a potential blood-based biomarker for screening cerebral amyloid deposition. Alzheimers Res. Ther. 9, 1-13. doi: 10.1186/s13195-017-0248-8

Pase, M. P., Beiser, A. S., Himali, J. J., Satizabal, C. L., Aparicio, H. J., DeCarli, C., et al. (2019). Assessment of plasma total tau level as a predictive biomarker for dementia and related endophenotypes. JAMA Neurol. 76:598. doi: 10.1001/jamaneurol.2018.4666

Pérez-Grijalba, V., Arbizu, J., Romero, J., Prieto, E., Pesini, P., Sarasa, L., et al. (2019). Plasma a $\beta 42 / 40$ ratio alone or combined with fdg-pet can accurately predict amyloid-pet positivity: a cross-sectional analysis from the ab255 study. Alzheimers Res Ther. 11, 1-19. doi: 10.1186/s13195-019-0549-1

Petzold, A. (2005). Neurofilament phosphoforms: surrogate markers for axonal injury, degeneration and loss. J. Neurol. Sci. 233, 183-198. doi: 10.1016/j.jns.2005.03.015

Qu, Y., Ma, Y., Huang, Y., Ou, Y., Shen, X., Chen, S., et al. (2021). Blood biomarkers for the diagnosis of amnestic mild cognitive impairment and Alzheimer's disease: a systematic review and meta-analysis. Neurosci. Biobehav. Rev. 128, 479-486. doi: 10.1016/j.neubiorev.2021.07.007

Quiroz, Y. T., Zetterberg, H., Reiman, E. M., Chen, Y., Su, Y., Fox-Fuller, J. T., et al. (2020). Plasma neurofilament light chain in the presenilin 1 e280a autosomal dominant Alzheimer's disease kindred: a cross-sectional and longitudinal cohort study. Lancet Neurol. 19, 513-521. doi: 10.1016/S1474-4422(20)30137-X

Roberts, K. F., Elbert, D. L., Kasten, T. P., Patterson, B. W., Sigurdson, W. C., Connors, R. E., et al. (2014). Amyloid-beta efflux from the central nervous system into the plasma. Ann. Neurol. 76, 837-844. doi: 10.1002/ana.24270

Schauer, S. P., Mylott, W. R., Yuan, M., Jenkins, R. G., Rodney Mathews, W., Honigberg, L. A., et al. (2018). Preanalytical approaches to improve recovery of amyloid- $\beta$ peptides from csf as measured by immunological or mass spectrometry-based assays. Alzheimers Res. Ther. 10, 1-16. doi: 10.1186/s13195-018-0445-0

Schindler, S. E., Bollinger, J. G., Ovod, V., Mawuenyega, K. G., Li, Y., Gordon, B. A., et al. (2019). High-precision plasma $\beta$-amyloid $42 / 40$ predicts current and future brain amyloidosis. Neurology 93, e1647-e1659. doi: 10.1212/WNL.0000000000008081

Schraen-Maschke, S., Sergeant, N., Dhaenens, C., Bombois, S., Deramecourt, V., Caillet-Boudin, M., et al. (2008). Tau as a biomarker of neurodegenerative diseases. Biomark. Med. 2, 363-384. doi: 10.2217/17520363.2.4.363

Shi, Y., Lu, X., Zhang, L., Shu, H., Gu, L., Wang, Z., et al. (2019). Potential value of plasma amyloid-beta, total tau, and neurofilament light for identification of early Alzheimer's disease. ACS Chem. Neurosci. 10, 3479-3485. doi: 10.1021/acschemneuro.9b00095

Sparks, D. L., Kryscio, R. J., Sabbagh, M. N., Ziolkowski, C., Lin, Y., Sparks, L. M., et al. (2012). Tau is reduced in ad plasma and validation of employed ELISA methods. Am. J. Neurodegener. Dis. 1, -106

Teunissen, C. E., Chiu, M. J., Yang, C. C., Yang, S. Y., Scheltens, P., Zetterberg, H., et al. (2018). Plasma amyloid-beta (abeta42) correlates with cerebrospinal fluid abeta42 in Alzheimer's disease. J. Alzheimers Dis. 62, 1857-1863. doi: 10.3233/JAD-170784
Teunissen, C. E., Petzold, A., Bennett, J. L., Berven, F. S., Brundin, L., Comabella, M., et al. (2009). A consensus protocol for the standardization of cerebrospinal fluid collection and biobanking. Neurology 73, 1914-1922. doi: 10.1212/WNL.0b013e3181c47cc2

Thijssen, E. H., La Joie, R., Wolf, A., Strom, A., Wang, P., Iaccarino, L., et al. (2020). Diagnostic value of plasma phosphorylated tau181 in Alzheimer's disease and frontotemporal lobar degeneration. Nat. Med. 26, 387-397. doi: 10.1038/s41591-020-0762-2

Tosun, D., Veitch, D., Aisen, P., Jack, C. R., Jagust, W. J., Petersen, R. C., et al. (2021). Detection of $\beta$-amyloid positivity in Alzheimer's disease neuroimaging initiative participants with demographics, cognition, MRI and plasma biomarkers. Brain Commun. 3:b8. doi: 10.1093/braincomms/ fcab008

Verberk, I. M. W., Slot, R. E., Verfaillie, S. C. J., Heijst, H., Prins, N. D., van Berckel, B. N. M., et al. (2018). Plasma amyloid as prescreener for the earliest Alzheimer pathological changes. Ann. Neurol. 84, 648-658. doi: 10.1002/ana. 25334

Vergallo, A., Mégret, L., Lista, S., Cavedo, E., Zetterberg, H., Blennow, K. et al. (2019). Plasma amyloid $\beta$ 40/42 ratio predicts cerebral amyloidosis in cognitively normal individuals at risk for Alzheimer's disease. Alzheimers Dement. 15, 764-775. doi: 10.1016/j.jalz.2019.03.009

Weston, P., Poole, T., Ryan, N. S., Nair, A., Liang, Y., Macpherson, K., et al. (2017). Serum neurofilament light in familial Alzheimer disease: a marker of early neurodegeneration. Neurology $89,2167-2175$. doi: 10.1212/WNL.0000000000004667

Wilke, C., Preische, O., Deuschle, C., Roeben, B., Apel, A., Barro, C., et al. (2016). Neurofilament light chain in $\mathrm{ftd}$ is elevated not only in cerebrospinal fluid, but also in serum. J. Neurol. Neurosurg. Psychiatry 87, 1270-1272. doi: 10.1136/jnnp-2015-312972

Xiao, Z., Wu, X., Wu, W., Yi, J., Liang, X., Ding, S., et al. (2021). Plasma biomarker profiles and the correlation with cognitive function across the clinical spectrum of Alzheimer's disease. Alzheimers Res. Ther. 13:123. doi: 10.1186/s13195-021-00864-x

Zetterberg, H., Skillbäck, T., Mattsson, N., Trojanowski, J. Q., Portelius, E., Shaw, L. M., et al. (2016). Association of cerebrospinal fluid neurofilament light concentration with Alzheimer disease progression. JAMA Neurol. 73:60. doi: 10.1001/jamaneurol.2015.3037

Conflict of Interest: The authors declare that the research was conducted in the absence of any commercial or financial relationships that could be construed as a potential conflict of interest.

Publisher's Note: All claims expressed in this article are solely those of the authors and do not necessarily represent those of their affiliated organizations, or those of the publisher, the editors and the reviewers. Any product that may be evaluated in this article, or claim that may be made by its manufacturer, is not guaranteed or endorsed by the publisher.

Copyright (๑) 2021 Jiao, Liu, Guo, Liao, Zhou, Weng, Xiao, Zhou, Wang, Jiang, Yang, Zhu, Zhou, Zhang, Wang, Yan, Tang and Shen. This is an open-access article distributed under the terms of the Creative Commons Attribution License (CC BY). The use, distribution or reproduction in other forums is permitted, provided the original author(s) and the copyright owner(s) are credited and that the original publication in this journal is cited, in accordance with accepted academic practice. No use, distribution or reproduction is permitted which does not comply with these terms. 